\title{
3D MODEL OF LIMESTONE INCLUSIONS IN MARITSA IZTOK MINE BASED ON ELECTRICAL RESISTIVITY TOMOGRAPHY
}

\author{
DOI: https://doi.org/10.18509/AGB.2017.06 \\ UDC: 550.837:004.925.83]:622.33'113(497.2) \\ Maya Grigorova ${ }^{1}$, Ivaylo Koprev ${ }^{2}$ \\ ${ }^{1}$ University of Mining and Geology “St. Ivan Rilski”, Bulgaria \\ Corresponding author: maya.grigorova86@gmail.com
}

Submitted: January 2017, Accepted: May 2017, Published: August 2017

\begin{abstract}
The 2D electrical resistivity tomography method (ERT) is a technique that can detect layers and geological features by exploiting resistivity contrasts between different layers using electrical current. This technique is useful for clearly determined resistivity boundaries, but limited where highly irregular or complex geological features such as inhomogeneous inclusions are present. In such a case 3D survey maybe required for inclusions type definition and also their size and location. The presented study demonstrates the efficiency of the ERT to map boundaries between distinct lithologies and detection of different inclusions on resistivity cross-sections.
\end{abstract}

Key words: Electrical resistivity tomography (ERT), limestone inclusions;

\section{Introduction}

The purpose of electrical resistivity surveys is to determine the subsurface resistivity distribution by making measurements on the ground surface. From these measurements, the true resistivity of the subsurface can be estimated. The ground resistivity is related to various geological parameters such as mineral and fluid content, porosity and degree of water saturation in the rock [1].

Since all geological structures are 3D in nature, a fully $3 \mathrm{D}$ resistivity survey using a $3 \mathrm{D}$ interpretation model should in theory give the most accurate results.

The present study helps in understanding the geological conditions in opencast Troyanovo-3 in the Maritsa Iztok Mine. Troyanovo-3 mine has form of a rectangle with west-east orientation and is located in the southern part of the Maritsa Iztok Basin. Extraction conditions in the opencast Troyanovo-3 become harder because of presence of solid limestone inclusions. This solid limestone bodies make extraction operations extremely challenging.

This study is focusing on opencast coal exploration in the area of Mini Maritsa Iztok
EAD. The usefulness of geophysical methods in mapping the hard rock inclusions in the overburden of the lignite mines is outlined.

\section{Description of the technology and data processing}

It has become increasingly popular in large scale mineral exploration surveys to use noninvasive geophysical methods for collecting more accurate information about location and geological properties of the ore bodies and surrounding area.

One of the most suitable methods for limestone inclusion detection is electrical resistivity tomography method (ERT). The resistivity method is one of the oldest geophysical survey techniques [2]. Resistivity measurements are based on the difference in resistivity between different sub-surface materials [3]. Electrical resistivity tomography (ERT) is a geophysical technique for imaging sub-surface structures based on electrical resistivity measurements made on the surface. The aim of the electrical resistivity tomography (ERT) technique is to scan the subsurface along the survey line using a selected electrode array [4]. In electrical re- 
sistivity tomography method resistivity changes in the vertical direction, as well as in the horizontal direction along the survey line.

2D ERT is done by 7 profiles (six of them with a length of $92 \mathrm{~m}$, and the ERT -7 with a length of $115 \mathrm{~m})$, connected to a multi-core cable, having a total length of $667 \mathrm{~m}$. A laptop microcomputer together with an electronic switching unit is used to automatically select the relevant four electrodes for each measurement. ERT data in this investigation were rapidly collected with an automated multi-electrode resistivity meter Terrameter SAS 1000. ERT profiles consist of a modeled cross-sectional (2D) plot of resistivity $(\Omega \mathrm{m})$ versus depth.

Preliminary data processing is based on determination of a two-dimensional (2D) model of the subsurface, which is then compared to the observed data and revised. ERT data processing and modeling were done using the RES2DINV. This program automatically determines the $2 \mathrm{D}$ resistivity model for the data obtained [5]. The 2D method of presenting resistivity data is limited where highly irregular or complex geological features are present and a 3D survey is required. Many 3D field data sets are constructed by combining a series of parallel 2D survey lines. Ideally the distance between the lines should be about the same as the distance between adjacent electrodes in each line. However, in most cases, the distance between the lines can be two or more times larger than the inter-electrode spacing along the lines. The data from each 2D survey line is initially inverted independently to give a series of 2D cross-sections. The 3D data set consists of a number of parallel 2D lines. Any point of the field corresponds to stratigraphic descriptions presented as pseudo wells.

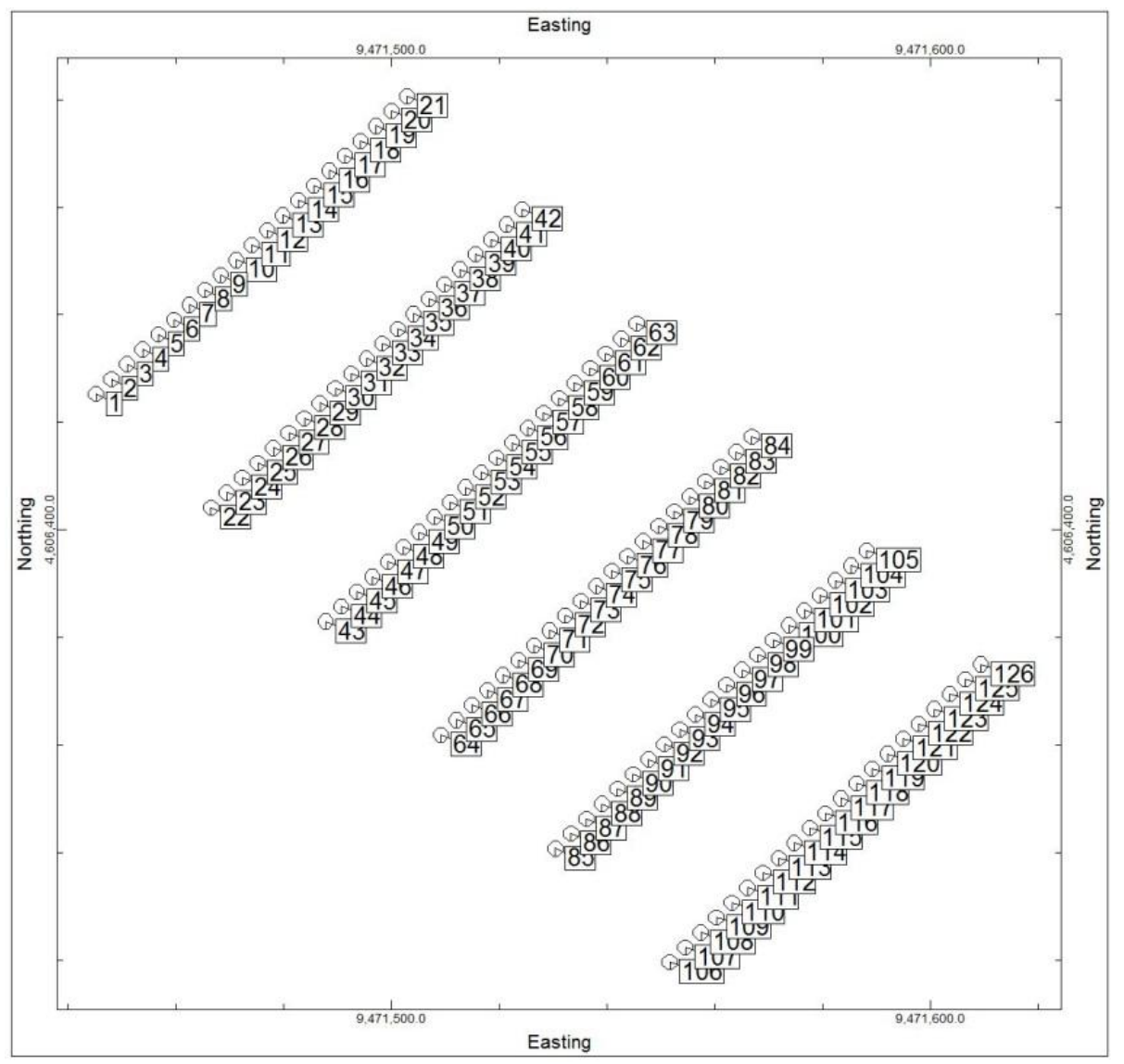

Figure 1: Series of 2D cross-sections at any point of which correspond stratigraphic descriptions presented as pseudo wells

The measured apparent resistivity values from all lines can be combined into a 3D data set and inverted with RockWorks 16 to give a $3 \mathrm{D}$ picture. The program is designed for geological data management, analysis and visualization. The quality of the $3 \mathrm{D}$ model is expected to be poorer than that produced with a complete $3 \mathrm{D}$ survey, but it 
is sufficient for supporting decision making process.

In some cases, measurements are made only in one direction. In the interpretation of data from $2 D$ resistivity imaging surveys, it is assumed that the subsurface geology does not change significantly in the direction that is perpendicular to the survey line. In areas with very complex geology, there are significant variations in the subsurface resistivity in this direction (i.e. the geology is 3D), which could cause distortions in the lower sections of the 2D model obtained. For this reason 3D data set was constructed by collecting data from parallel 2D survey lines.

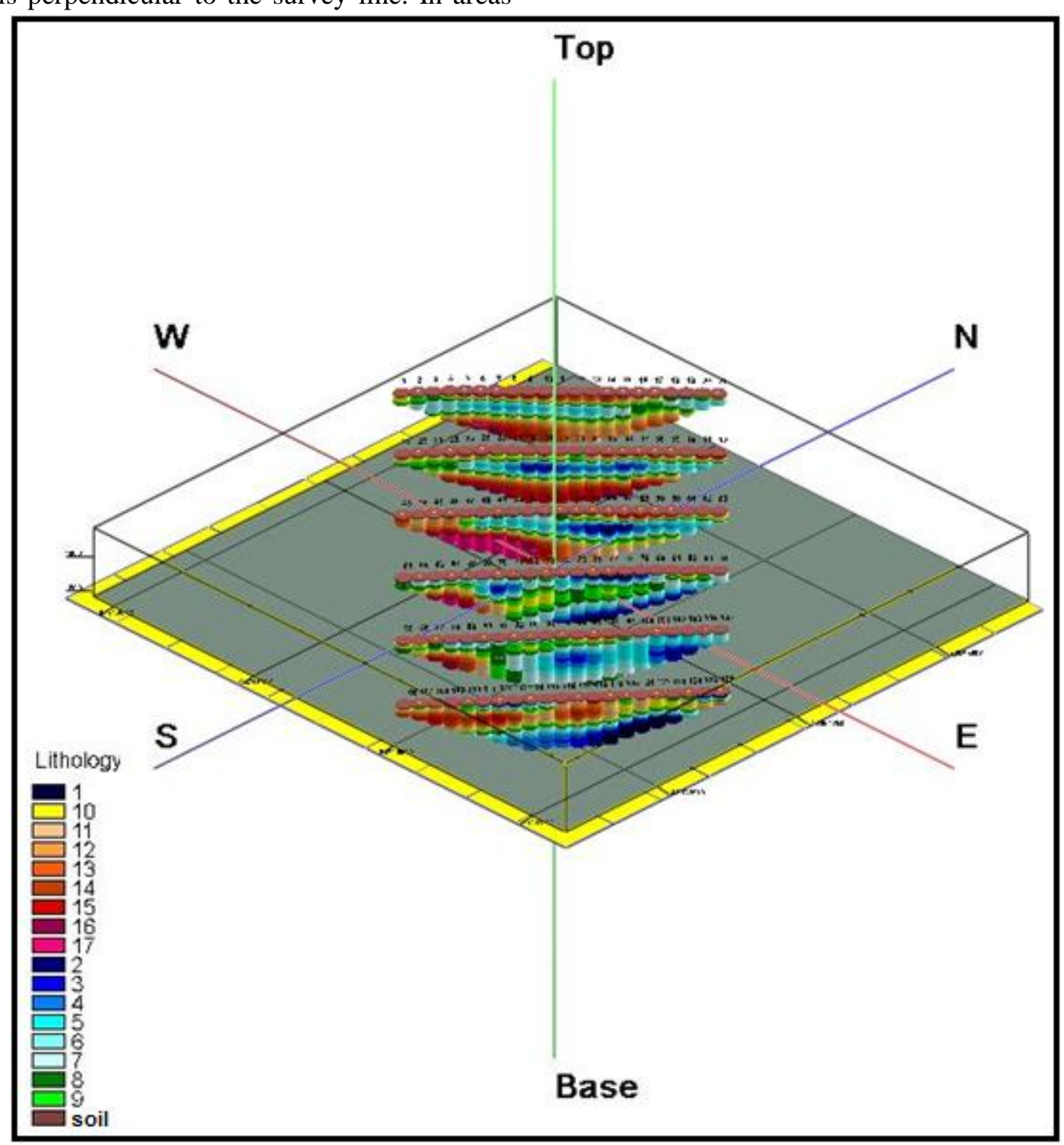

Figure 2: Field measurements situation plan of 2D cross-sections with pseudo wells

From studies conducted, 17 areas of changes in the electrical resistivity values were established. The electrical resistivity varies from 10 to $50 \Omega \mathrm{m}$. Part of these values present different lithological varieties. The aim of this study is to divide this lithological varieties so that to collect an information about the presence, location and characteristics of the solid limestone bodies incorporated in the mostly clay medium.

\section{Results and interpretation}

Geological materials have resistivity values that enable identification of boundaries between distinct lithologies on resistivity cross-sections. 
In Figure 3 are presented 3D block models of the different lithological variations of the studied area which represent electrical resistivity of the medium that build geological section. The subsurface is known to be highly inhomogeneous consisting of sandy clays and carbonated sand with carbonate and limestone inclusions.

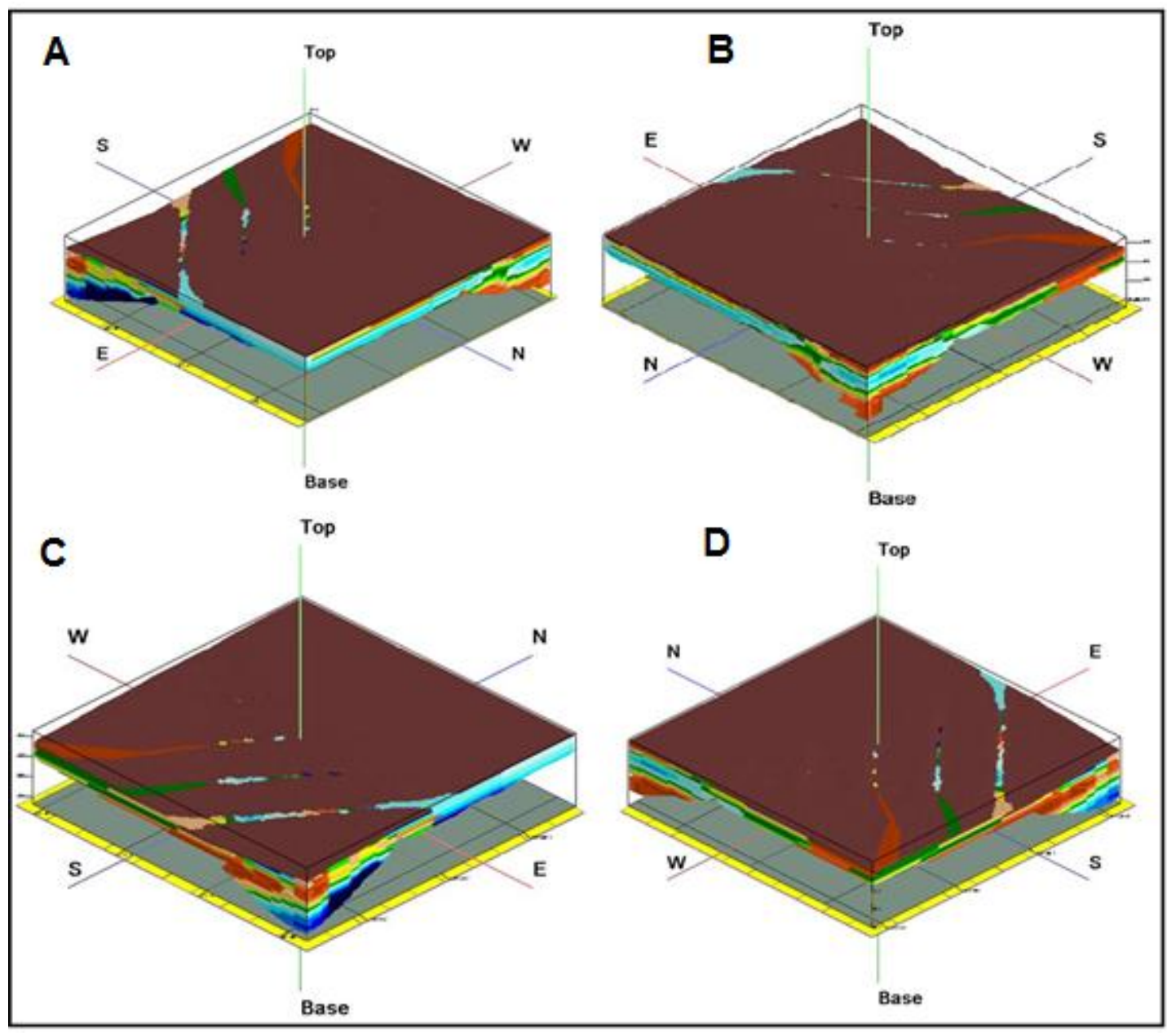

Figure 3: 3D block models of different lithological variations of the studied area:

$$
\text { A - East-North, B - North-West, C - South - East, D - West-South }
$$

Based on the obtained block models the following results for the studied geological varieties were obtain (Figure 4):

- Soil;

- Lithology 1 to 8-Sandy clays with single limestone inclusions;

- Lithology 9 to 11-Sandy clays with limestone inclusions;

- Lithology 12 to 14-Untenable limestone layers;
- Lithology 15 to 17-Carbonated sand with carbonate inclusions.

Also based on the same block models different lithological varieties can be presented separately as quantity volumes. This information helps in understanding the distribution and location of different lithologies presented in this area. 




Figure 4: 3D models of the different lithological variations 


\section{Conclusion}

2D and 3D resistivity imaging methods are simple, fast, inexpensive, and relatively accurate techniques used in geophysical exploration. In this study, 2D and $3 \mathrm{D}$ resistivity imaging methods are used to produce images of the subsurface structure of the MaritzaIztok Mine EAD. The result of presented inverse model shows that the subsurface resistivity distribution in this area is highly inhomogeneous and can change rapidly within a short distance. Generally, this study revealed lithologies composed mostly of soil, sand, sandstone, sandy clay, clay and untenable limestone layers and limestone inclusions to a depth of about 12-14 $\mathrm{m}$. Results provided useful information about the resistivity distribution beneath the studied area and helps in decision making process for location of blasting boreholes, which has to be arrange in the process of solid limestone inclusions fragmentation.

\section{Reference}

[1]Loke, M.H., Tutorial: 2-D and 3-D electrical imaging surveys, 2011.

[2]Loke, M.H., Electrical resistivity surveys and data interpretation. In Gupta, H (ed.), Solid Earth Geophysics Encyclopedia (2nd Edition), "Electrical \& Electromagnetic", SpringerVerlag, 276-283, 2011.

[3]Friedel, S., Thielen, A. \& Springman, S.M., Investigation of a slope endangeredby rainfallinduced landslides using 3D resistivity tomography and geotechnicaltesting. Journal of Applied Geophysics, 60, 100-114, 2006.

[4]Drahor, M.G., Integrated geophysical studies in the upper part of Sardis archaeological site, Turkey: Journal of Applied Geophysics, 59 (3), 205-223, 2006.

[5] Griffiths, D.H., Barker, R.D., "Two Dimensional Resistivity Imaging and Modeling in areas of Complex Geology", Journal of Applied Geophysics, (29) 211-226, 1993. 